\title{
definiciones y métodos de medida de las características geométricas de las barras corrugadas de acero, para uso del sello CIETSID
}

CARLOS BRIZ PINDAO, ingeniero industrial GREGORIO TABERA SECO, ingeniero técnico

$458-3$

\section{sinopsis}

El presente trabajo ha tenido como finalidad principal, tanto la aclaración de las definiciones de cada una de las medidas de las características geométricas principales de las barras de acero corrugado, como la descripción del método de medida de las mismas y de los aparatos más idóneos para la realización de cada una de ellas.

Todos estos aparatos han sido reunidos en un solo montaje creado por los autores del presente trabajo, al objeto de mecanizar en lo posible la realización de las medidas indicadas, de manera fácil, comparabie y a la vez económíca, cuya descripción y modo de empleo se describen detalladamente en él.

\section{INTRODUCCION}

Debido a las diferentes formas de medir las características geométricas de las barras corrugadas de acero y al empleo de aparatos de medida diversos, se comprende fácilmente la necesidad de unificar tanto el método de medida como los aparatos más idóneos para la realización de cada una de ellas.

Asimismo, tampoco están definidas claramente estas medidas en su concepto, comprendiéndose fácilmente la necesidad de tal definición como paso previo al proceso de medida. A la vista de esta situación, comprobada en la práctica por los inspectores del Sello de Conformidad CIETSID, en las visitas realizadas a factorias hasta la fecha, y al objeto de unificar en lo posible tanto los aparatos de medida como el método de realización de las mismas y, a la vez, aclarar la definición de cada una de ellas, la Secretaría del Sello de Conformidad CIETSID ha redactado el presente método de medida, en el cual incluye las definiciones de las características geométricas de las barras corrugadas, exigidas por el Sello, así como el método y aparatos más idóneos para la realización de su medición.

También se incluye la descripción del montaje efectuado por la Secretaría de varios aparatos de medida, al que denomina "montaje 'Brizta' para la medida de las características geométricas de los corrugados", creado simplemente con objeto de mecanizar en lo posible la realización de estas medidas, de manera que resulte fácil, comparable, y a la vez económica, dada la sencillez de dicho montaje. Naturalmente, el método que se describe es totalmente válido para ser llevado a cabo con un proyector de perfiles, si bien hay que comprender que la diferencia de precio entre ambos aparatos es tal que muchas empresas no podrán permitirse la adquisición de este último, pudiendo, en cambio, valerse perfectamente del que se describe en este método. 


\section{DEFINICIONES}

MEDIDAS

1. Altura máxima de corruga.

2. Separación de corrugas.

3. Paso de hélice.

4. Inclinación de corrugas.
SIMBOLO

a

Distancia entre el punto más alto de la corruga y el núcleo de la barra, medida en dirección normal al eje de la barra y en el plano que contenga a dicho eje (ver apartado 4.1).

Distancia entre puntos homólogos de dos corrugas consecutivas, medida en dirección paralela al eje de la barra (ver apartado 4.2).

Distancia entre puntos homólogos consecutivos situados en la misma generatriz (ver apartado 4.3).

Angulo de la corruga con el eje de la barra.

\section{DESCRIPCION DEL MONTAJE "BRIZTA» (foto 1)}

Este montaje, diseñado esencialmente para la medida de las características geométricas de las corrugas de los aceros, para armaduras de hormigón, de una manera mecánico-manual, consta de los siguientes elementos:

a) Carcasa soporte, de material ligero, resistente (madera, plástico, aluminio, etc.).

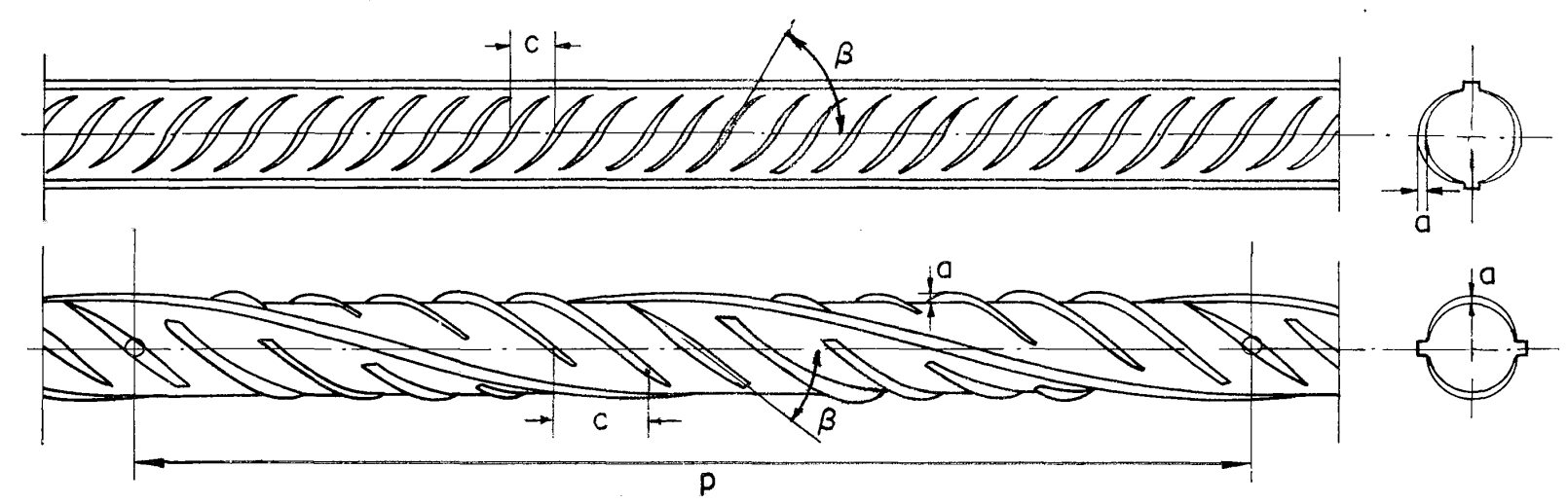

CROQUIS ORIENTATIVO 


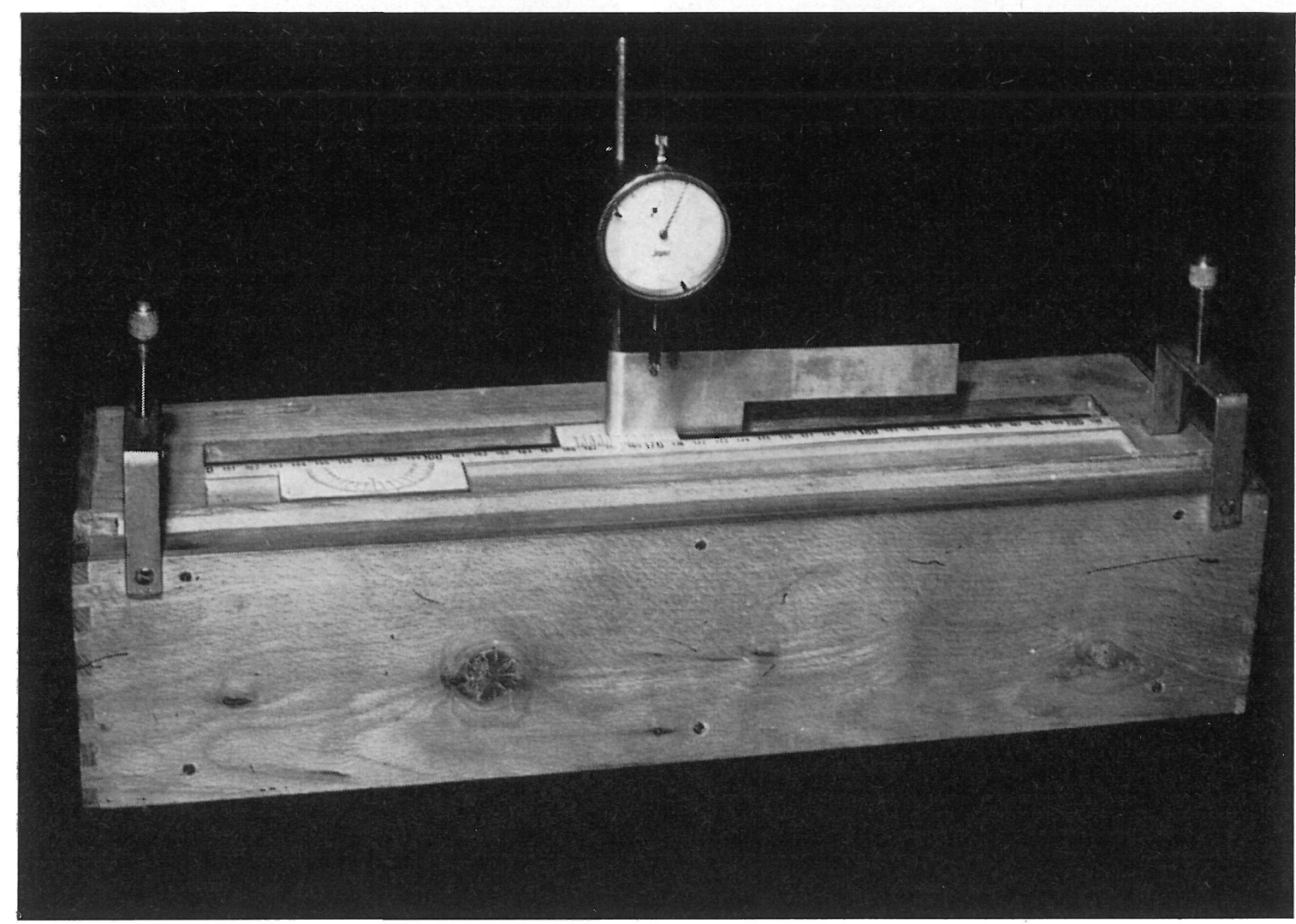

Vista general del montaje «Brizta».

En su interior va acoplado el tornillo sinfín, que permitirá el desplazamiento del carro porta-comparador, y una guía metálica para dicho carro.

En el exterior, y en su costado derecho, lleva una manivela unida al sinfín, para su accionamiento; en la parte superior va provisto de las siguientes partes: entalla longitudinal en V, para apoyo de las barras; entalla longitudinal en U, para deslizamiento del semicírculo graduado; regleta graduada; acanaladura longitudinal, para permitir el movimiento del carro, y abrazaderas para sujeción de la barra.

b) Carro porta-comparador cuya base desliza sobre la guía metálica y a la cual va adosado un casquillo roscado interiormente, en el cual va acoplado el sinfín para el movimiento del carro. En la parte exterior de su base va acoplado un nonius para precisar las medidas realizadas con la regleta graduada, y un eje vertical, soporte del comparador. En este eje lleva acoplado un índice giratorio para la determinación de ángulos de corrugas.

c) Semicírculo graduado, situado sobre una pieza de material resistente, cuya cara inferior esté lo suficientemente pulida para permitir su deslizamiento en la entalla correspondiente.

d) Comparador con apreciación de $0,01 \mathrm{~mm}$, sujeto al eje vertical mediante una pieza que permite su desplazamiento en sentido vertical a lo largo del eje y horizontal en el sentido de dicha pieza, mediante los cuales podrá situarse el palpador centrado en el vértice de la entalla y reposando con la presión apropiada sobre cada barra a medir. 

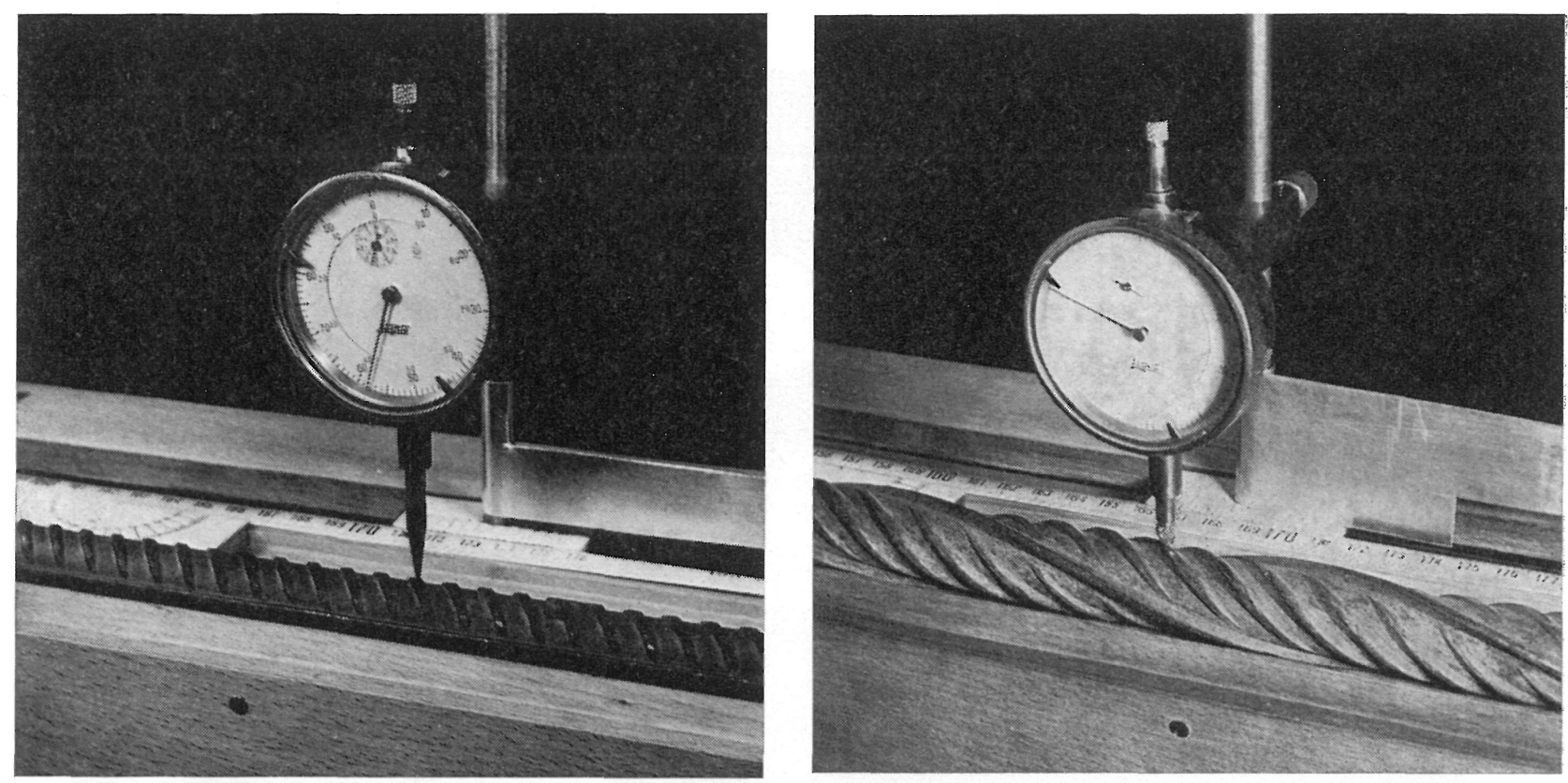

\section{MEDIDAS. REALIZACION}

\subsection{Altura máxima de corruga}

Se medirá con el "Brizta". La barra se situará en la entalla del aparato de forma que el palpador del comparador repose sobre una de las corrugas, ejerciendo sobre ella una ligera presión, girando y desplazando a continuación la barra ligeramente en ambos sentidos, de manera que el palpador deslice sobre la corruga, hasta encontrarse el punto más alto de dicha corruga, por medio del comparador (fotos 2 y 2'), fijándose a continuación con las abrazaderas.

3

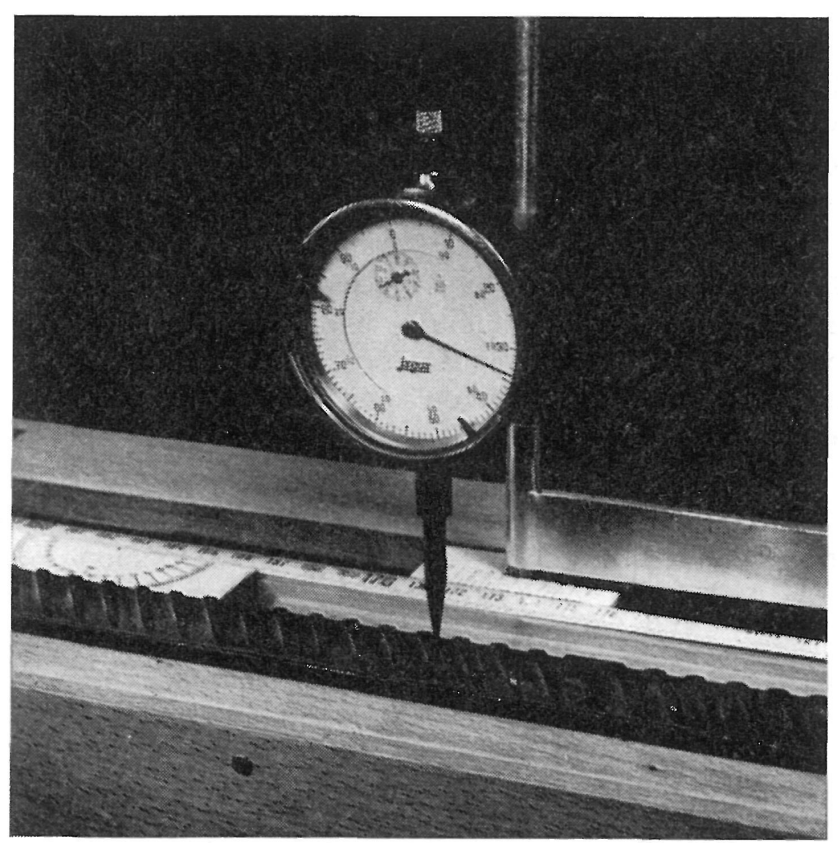

Altura de corruga (medida en el núcleo).

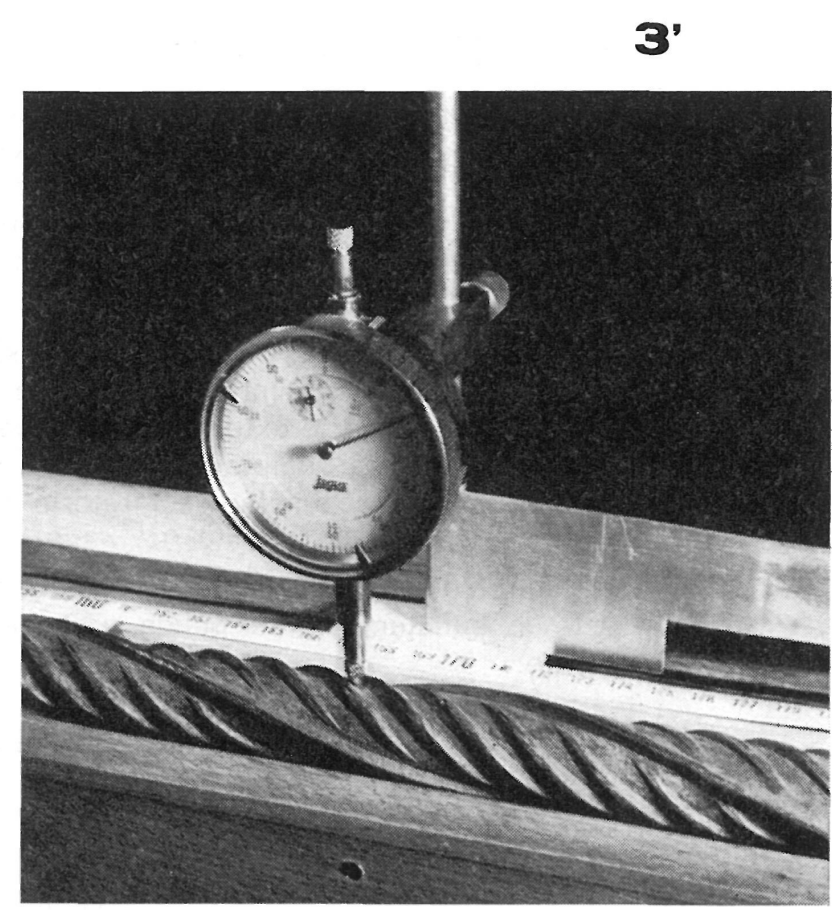


En este punto se anotará la lectura del comparador.

Seguidamente, y mediante el sinfín del aparato, se desplazará el carro soporte del comparador en dirección paralela al eje de la barra, hasta que el palpador apoye sobre el núcleo de la misma, anotándose en ese momento la lectura del comparador (fotos 3 y $3^{\prime}$ ).

Esta operación y lectura se realizarán en ambos lados de la corruga.

La diferencia entre las medidas obtenidas al apoyar el palpador en el núcleo y en la corruga, dará como resultado la altura de la corruga, promediándose los valores obtenidos a ambos lados de la misma.

Se realizará esta medida, como mínimo, en tres corrugas elegidas al azar, promediándose los valores obtenidos.

\subsection{Separación de corrugas}

Se medirá con el "Brizta". Situada la barra en el aparato, según se ha descrito en el apartado 4.1, y el palpador en un punto de la corruga, se anotará la lectura indicada en la regleta graduada (foto 4 ).

A continuación se desplazará el carro soporte del comparador, mediante el sinfín, en dirección paralela al eje de la barra, hasta que el palpador apoye sobre el punto homólogo del anterior, de otra corruga situada respecto a la primera, de acuerdo con los siguientes criterios:

a) Aceros de dureza natural: de forma que se incluyan

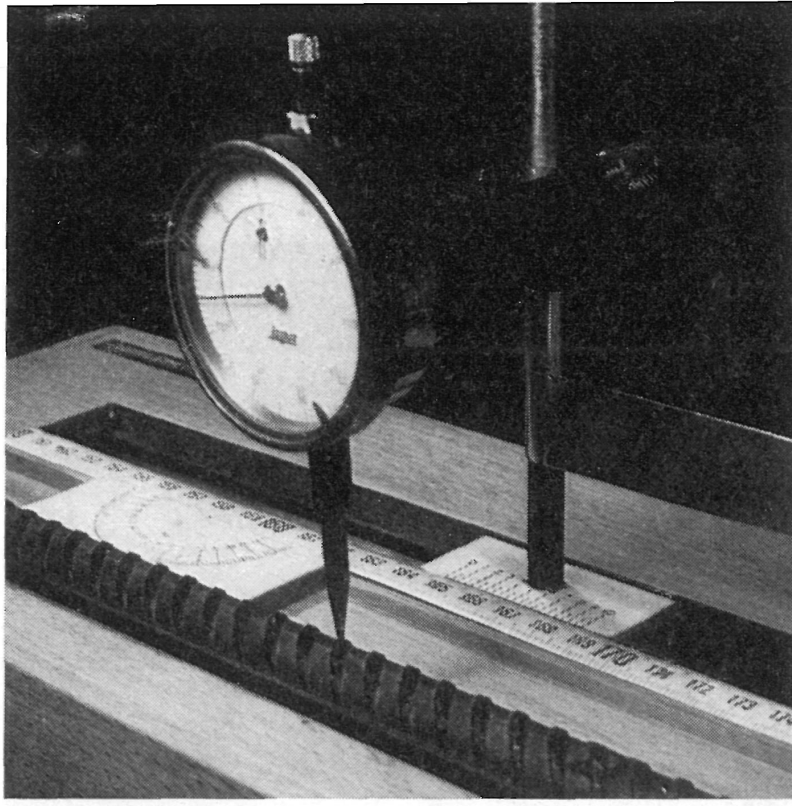

Separación entre corrugas (medida en la primera corruga de referencia).

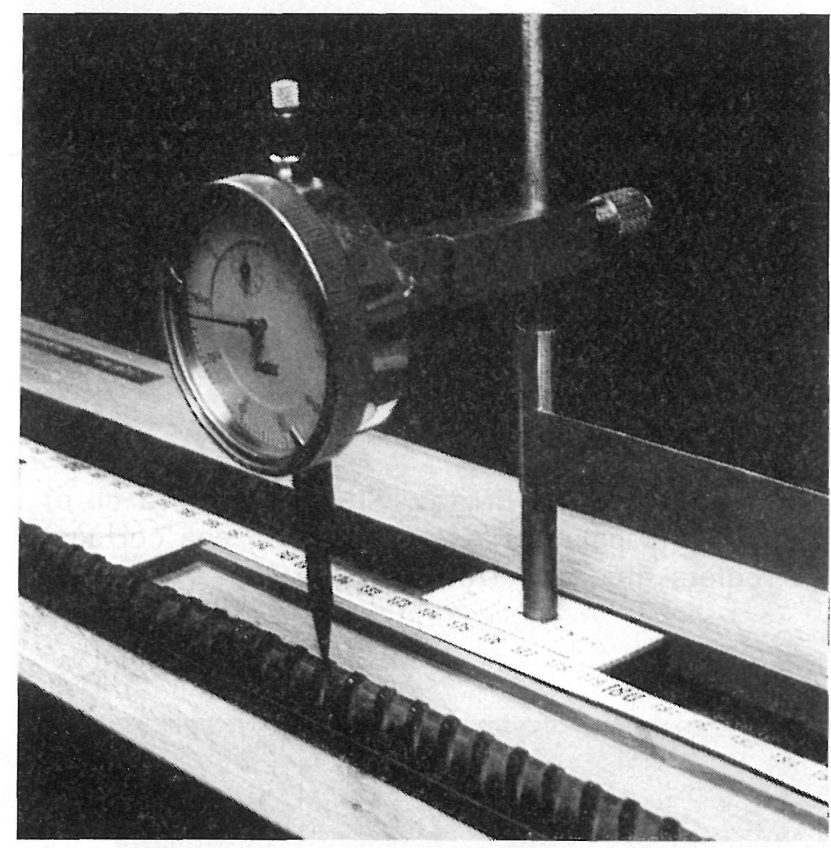

5

Paso de hélice (primera medida de referencia).

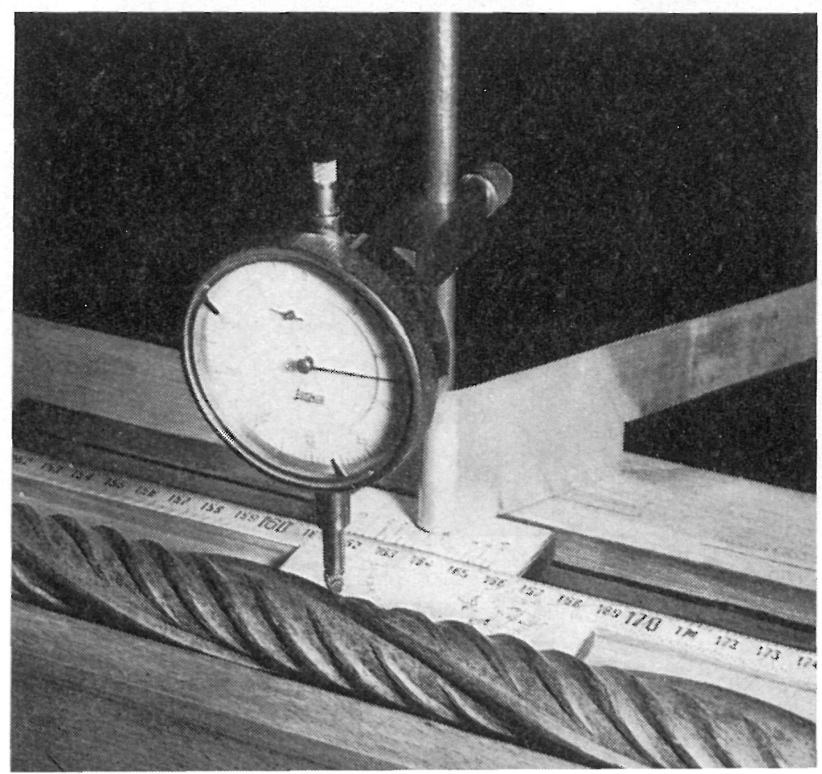




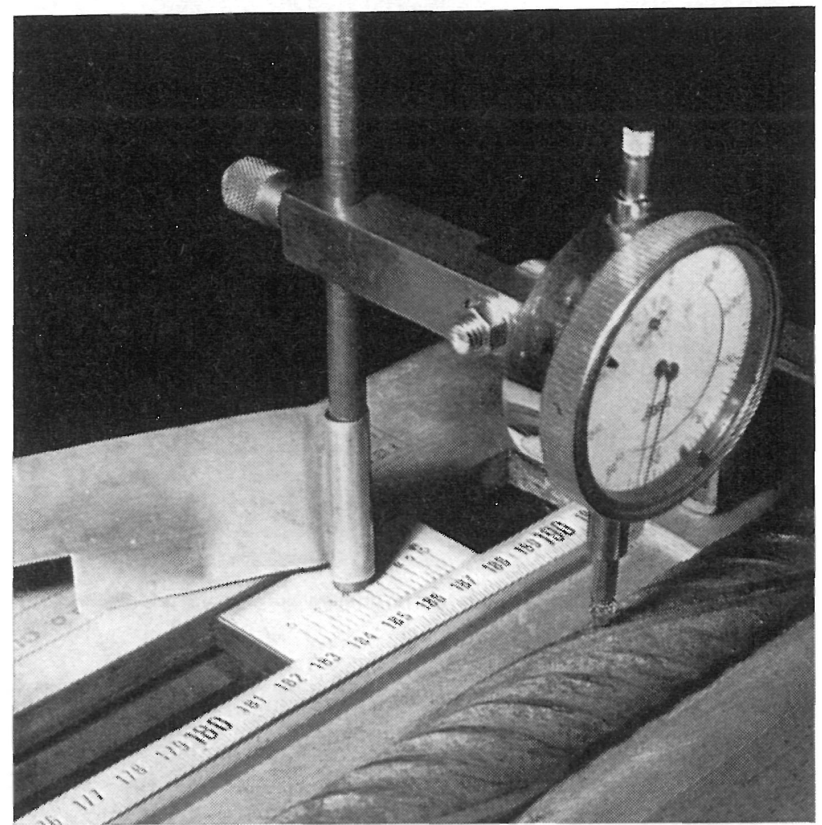

Paso de hélice (segunda medida de referencia). diez intervalos consecutivos entre ambas corrugas de medida (foto 5).

b) Aceros deformados en frío: de forma que esta segunda corruga coincida en posición relativa con la primera y esté situada sobre la misma hélice que aquélla.

Una vez situado el palpador en la segunda corruga de referencia, se anotará la lectura indicada en la regleta graduada, calculándose por diferencia con la primitiva la separación correspondiente al número de intervalos elegido para el caso a) y al que resulte según el tipo de dibujo, entre ambas corrugas, a lo largo de su hélice, para el caso b).

Para determinar la separación de corrugas se dividirá esta diferencia entre 10 para los aceros de dureza natural, y entre el número de intervalos resultante, antes mencionado, para los aceros deformados en frío.

\subsection{Paso de hélice}

Se medirá con el "Brizta". Situada la barra en el aparato, según se ha descrito en el apartado 4.1, y el palpador en un punto de una corruga o cordón, se anotará la lectura indicada en la regleta graduada (foto 6 ).

8

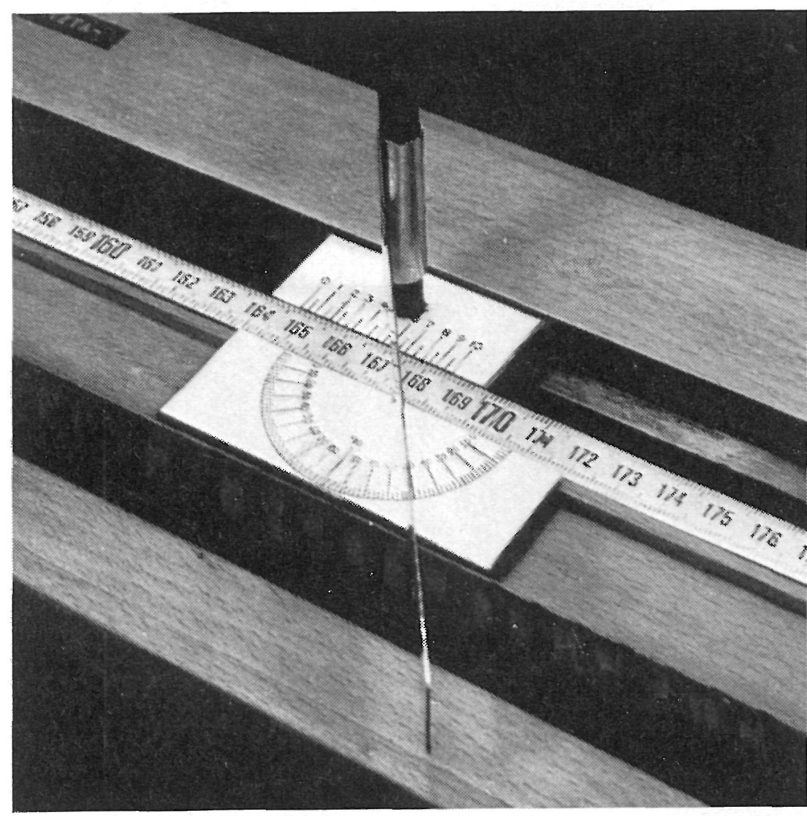

Inclinación de corruga. $\mathbf{8}^{\prime}$

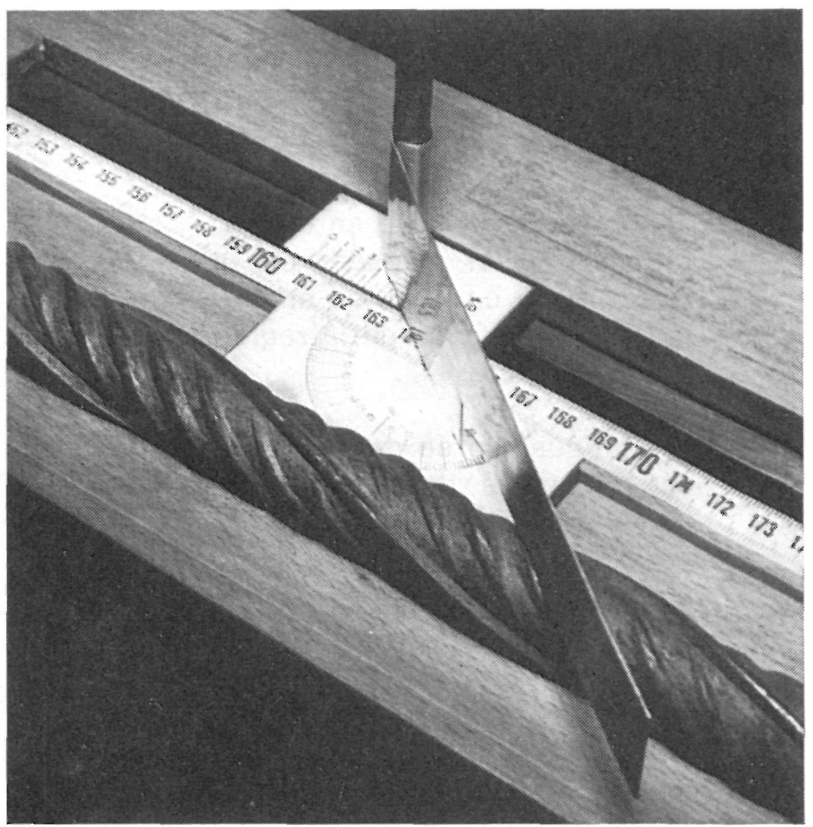


Seguidamente, se desplazará el carro soporte del comparador, mediante el sinfín, en dirección paralela al eje de la barra hasta que el palpador apoye sobre el punto homólogo del anterior, de una corruga o cordón, situada en la misma posición relativa que la primera y sobre la misma hélice, anotándose la lectura indicada en la regleta graduada (foto 7).

La diferencia entre ambas lecturas dará como resultado el paso de hélice.

\subsection{Inclinación de corruga}

Se medirá con el "Brizta". Situada la barra según se indicó en el apartado 4.1, se girará el índice situado en el eje del carro soporte hasta que quede paralelo a la corruga en su zona de máxima altura, desplazando a continuación el semicírculo graduado hasta que su origen coincida con el eje del índice, leyéndose entonces directamente el ángulo de inclinación de las corrugas (fotos 8 y $8^{\prime}$ ).

NOTA: Además del montaje "Brizta» y proyector de perfiles, podrán utilizarse para la realización de las medidas de las características geométricas de los corrugados, aparatos de precisión manejados separadamente, tales como: calibre pie de rey, comparador con apreciación de $0,01 \mathrm{~mm}$, regla graduada, etc., siempre y cuando el procedimiento de medida se adapte al descrito anteriormente.

\section{résumé}

Définitions et méthodes de mesure des caractéristiques géométriques des barres en acier crénelé, pour l'application du sceau CIETSID

Carlos Briz Pindao, ingénieur industriel Gregorio Tabera Seco, ingénieur technique

Ce travail a eu pour but principal de préres des caractéristiques géométriques prin cipales des barres en acier crénélé et do décrire la méthode de mesure de celles-ci et des appareils les plus appropriés à la réalisation de chacune d'entre elles.

Tous ces appareils ont été réunis en un seul montage, créé par les auteurs de ce mesure du possible, d'une manière facile, comparable et a la fois économique, la réalisation de ces mesures.

La description des appareils et leur mode d'emploi complètent ce travail.

\section{summary}

Definitions and measuring methods of the geometrical characteristics of corrugated steel bars, for the use of the CIETSID seal

Carlos Briz Pindao, industrial engineer Gregorio Tabera Seco, industrial engineer

The main object of the present work has been both to clarify the definitions of every measure of the main geometrical characteristics of corrugated steel bars, as well as to describe the most suitable apparatus for

All these apparatus have been gathered in one single assembly, made by the authors of the present work, so as to mechanize the above mentioned measuring methods as tar as possible, as well as in a simple, al manner.

\section{zusammenfassung}

Definitionen und Messmethoden der Geometrischen Merkmale der Wellenstahlstäbe, für den CIETSID stempel

Carlos Briz Pindao, Gewerbeingenieur

Gregorio Tabera Seco, Gewerbeingenieur

Der Hauptzwerck der vorhandenen Arbeit ist der folgende gewesen: die Erläuterung der Definitionen jeden Masses der geometrischen Hauptmerkmale von Wellenstahistäben und weiterhin, die Beschreibung der Messmethoführen der Messungend messendsten Apparate.

Alle diese Apparate sind in eine einzige, von den Autoren der vorhandenen Arbeit geschaffenen Montage zusammengeführt worden, um das Aussuhren der oben erwähnten Messungen, so viel wie moglich und in zeitig oinonomischen weicharen und gleich- 